\title{
Funções executivas e regulação cognitivo-emocional: conexões anatômicas e funcionais
}

\author{
Funciones ejecutivas y regulación cognitivo-emocional: conexiones \\ anatómicas y funcionales
}

\section{Executive functions and cognitive-emotional regulation: anatomical and functional connections}

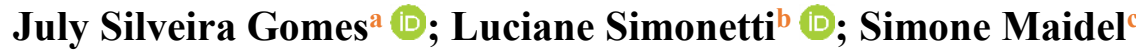

a Psicóloga e mestre em Psicologia Cognitiva pela Universidade Federal de Santa Catarina. Doutora em Psiquiatria e Psicologia Médica, pela Escola Paulista de Medicina, Universidade Federal de São Paulo - UNIFESP, São Paulo, SP, Brasil - E-mail: july.flp@gmail.com

\footnotetext{
${ }^{\text {b }}$ Psicóloga pelo Centro Universitário para o Desenvolvimento do Alto Vale do Itajaí/SC - UNIDAVI. Especialização em Terapia Cognitiva pelo Centro de Terapia Cognitiva - Veda. Mestre Especialista pelo Conselho Federal de Psicologia. Mestre em Ciências pelo Departamento de Psicobiologia da Universidade Federal de São Paulo - UNIFESP, São Paulo, SP, Brasil - E-mail: lucianesimonetti29@gmail.com

${ }^{c}$ Psicóloga, Doutora em Psicologia e Mestre em Neurociências pela Universidade Federal de Santa Catarina (UFSC). Especialista em Psicologia Clínica e em Gestalt-terapia. Professora do Instituto Federal do Maranhão - IFMA. Santa Inês, MA, Brasil - E-mail: simonemaidel@gmail.com
}

\begin{abstract}
Resumo: O estudo das funções executivas (FEs) tem sido alvo de pesquisas com foco na relação cérebro-comportamento, principalmente no que diz respeito ao desenvolvimento de habilidades sociais e o controle emocional em crianças, adolescentes e adultos com lesão cerebral no lobo frontal. Uma vez que essas funções são fundamentais para a adaptação do indivíduo em sociedade, é possível perceber sua importância na evolução filogenética do homem como um mecanismo de regulação e controle do comportamento. Por essa razão, este artigo tem como objetivo fornecer um panorama geral de estudos e descobertas acerca das FEs, focando nos aspectos neuroanatômicos e seus correspondentes comportamentais, especialmente nos processos cognitivos e na regulação emocional.
\end{abstract}

Palavras-chave: Funções executivas. Córtex pré-frontal. Regulação comportamental. Regulação emocional.

\begin{abstract}
Executive functions have being target in many studies focusing on the brain-behavior relationship, especially those involving the development of social skills and emotional control in children and teenagers, as well as in frontal lobe lesion in adults. Considering that these functions are fundamental for adaptation in society, its importance as a phylogenetic evolutionary mechanism for emotion regulation and control stands out. Taking this into account, this paper has the objective to build a general overview about executive functions from studies and findings presented by current literature, focusing in neuro-anatomical characteristics and its relation with behavior, especially in cognitive and emotional control.
\end{abstract}

Keywords: Executive Functions. Prefrontal córtex. Behavioral regulation. Emotional regulation.

Resumen: El estudio de las funciones ejecutivas (FE) ha sido objeto de investigaciones enfocadas en la relación cerebrocomportamiento, principalmente en lo que se refiere al desarrollo de habilidades sociales y el control emocional en niños, adolescentes y adultos con lesión cerebral en el lóbulo frontal. Una vez que esas funciones son fundamentales para la adaptación del individuo en obra, forneça um link para a licença, e indicar se foram feitas alterações. 
sociedad, es posible percibir su importancia en la evolución filogenética del hombre como un mecanismo de regulación y control del comportamiento. Por esta razón, este artículo tiene como objetivo proporcionar un panorama general de estudios y descubrimientos acerca de las FE, enfocándose en los aspectos neuroanatómicos y sus correspondientes comportamientos, especialmente en los procesos cognitivos y en la regulación emocional.

Palabras clave: Funciones ejecutivas. Corteza prefrontal. Regulación del comportamento. Regulación emocional.

\section{Como citar o artigo:}

GOMES, J. S; SIMONETTI, L; MAIDEL, S. Funções executivas e regulação cognitivo-emocional: conexões anatômicas e funcionais Revista de Ciências Humanas, Florianópolis, v. 52, 2018 DOI: 10.5007/21784582.2018.42170

Os avanços tecnológico e metodológico-científico observados ao longo das últimas décadas, principalmente nas áreas de neurociências e cognição, têm possibilitado uma maior compreensão acerca do papel das funções executivas (FEs) e da influência do córtex frontal sobre o comportamento humano, destacando sua importância para o desenvolvimento dos processos cognitivos superiores (ARDILA, 2008). De modo geral, as FEs são consideradas processos cognitivos de controle e regulação, responsáveis pela interação contínua entre mecanismos comportamentais automatizados (que implicam "baixo" processamento cognitivo) e comportamentos orientados para metas específicas (considerados de "alto" processamento cognitivo). Nesse sentido, as FEs estão associadas ao monitoramento de comportamentos para a realização de uma tarefa ou de um objetivo (CAPOVILLA, ASSEF, \& COZZA, 2007).

O processo regulatório ocorre por meio de habilidades cognitivas que incluem planejamento, monitoramento, flexibilidade, controle inibitório e regulação emocional (LEZAK, HOWIESON, \& LORING, 2004), sendo que FE é utilizado como um termo guarda-chuva, englobando essas funções (GOLDSTEIN ET AL., 2014). Três dessas FEs - atualização (updating), mudança (shiffting) e inibição (inhibition) - têm recebido maior destaque por diferentes grupos de pesquisadores, que vêm desenvolvendo instrumentos para avaliação fidedigna desses construtos (FOURNIER-VICENTE, LARIGAUDERIE, \& GAONAC'H, 2008; MIYAKE ET AL., 2000; MIYAKE, \& FRIEDMAN, 2012), como: Plus-Minus, Number-Letter e Local-Global, para tarefas de mudança; Keep Track, Tone Monitoring e Letter Memory, para tarefas de atualização; e Antisaccade, Stop-Signal e Stroop para tarefas de inibição.

Em geral, no contexto de resolução de problemas, as FEs se referem a todo processo de tomar uma decisão e executá-la por meio do controle consciente de estímulos em função de um objetivo ou problema a ser resolvido. Como componentes cognitivos dessa habilidade, verificam-se a capacidade de inibição e mudança de comportamento (durante a própria execução do mesmo), os processos de memória de trabalho (mais conhecida como memória operacional), a atenção sustentada e a atenção seletiva (ALVAREZ, \& EMORY, 2006). Ardila (2008) destaca como uma habilidade executiva a capacidade de filtrar estímulos intervenientes e antecipar consequências (seja das ações de outros ou 
das próprias ações), o que facilita a reformulação e ajuste de próprio comportamento durante a própria execução deste.

No que diz respeito às implicações na vida diária, pessoas que sofreram traumatismo cranioencefálico e que tiveram como consequência uma lesão no córtex frontal, geralmente apresentam alterações nas FEs. Tais alterações podem afetar desde as relações sociais e interpessoais até os processos cognitivos necessários para a funcionalidade e a adaptação do sujeito. Esses prejuízos podem ser observados na falta de organização e planejamento, na tomada de decisão prejudicada, na desinibição comportamental e até em alterações na personalidade e desregulação emocional (LEZAK, \& O’BRIEN, 1988; PRIGATANO, 1992).

Tendo em vista o papel das FEs para a regulação e o controle do comportamento humano, propõe-se tecer um panorama dos estudos sobre as FEs, focando os aspectos neuroanatômicos e seus correspondentes comportamentais, especialmente em termos de processos cognitivos e regulação emocional.

\section{O ESTUDO EXPERIMENTAL DAS FES}

Ao analisar-se a literatura focada na avaliação das FEs, duas linhas de abordagens podem ser observadas: uma delas objetiva avaliar aspectos puramente cognitivos, minimizando a influência de variáveis inerentes ao sujeito que possam interferir no resultado esperado; enquanto outras procuram analisar o papel ou influência dos aspectos emocionais no funcionamento executivo.

Na primeira abordagem, os estudos buscam mensurar aspectos puramente cognitivos, isolados de variáveis intervenientes, tendendo a focar nos aspectos metacognitivos destas funções. De modo geral, os experimentos utilizam tarefas que requeiram soluções lógicas por parte do sujeito, como os testes: Wisconsin Card Sorting Test (WCST - Teste de Wisconsin de Classificação de Cartas); Tower of London (Torre de Londres); Trail-Marking (Teste de Trilhas); Hayling Sentence Completion Test (Teste de Hayling); Phonemic Verbal Fluency (Teste de Fluência Verbal); Dimensional Change Card Sort (DCCS); e Stroop Color Word Interference Task (Teste de Stroop) (ALVAREZ, \& EMORY, 2006; ARDILA, 2008; GILBERT, \& BURGESS, 2008; ZELAZO, \& CUNNINGHAM, 2010).

Em comum, tais testes tendem a mobilizar processos cognitivos que exigem flexibilidade e que estão além da aprendizagem por associação estímulo-resposta. Além disso, tais tarefas exigem resolução de problemas e inibição de estímulos não relevantes ou a manipulação de informações, por um período de tempo, e a manutenção de respostas, muitas vezes com algum tipo de interferência cognitiva (ALVAREZ, \& EMORY, 2006; GILBERT, \& BURGESS, 2008). Outro aspecto comum é 
o fato de as tarefas serem emocionalmente neutras e seus objetivos voltados à própria tarefa, portanto, externos ao sujeito que as executa (ARDILA, 2008).

O valor ecológico de experimentos dessa natureza é baixo, tendo-se em vista que na vida diária as situações que exigem flexibilidade cognitiva não acontecem em um ambiente controlado, no qual é possível observar o fenômeno de forma isolada, como é realizado, por exemplo, na tarefa de Wisconsin. Nessa tarefa, o sujeito deve combinar cartas de um conjunto com quatro cartas-estímulo dispostas à sua frente, seguindo um critério a ser descoberto com base no feedback do examinador. Nesse teste, o sujeito tem oportunidade de mudar sua resposta com base nesse feedback, e o processo de flexibilidade cognitiva é inferido a partir da resposta emitida pelo sujeito (para mais esclarecimentos sobre essa tarefa, consultar: MIGUEL, 2005).

No entanto, em situações do dia-a-dia, os seres humanos atuam e fazem escolhas dentro de contextos permeados por afetos, emoções e motivações e, desse modo, esse aspecto não pode ser desconsiderado (ARDILA, 2008; BARKLEY, 2001). Zelazo (2004) denomina o componente emocional/ motivacional das FEs de "funções quentes" (em contraposição aos componentes metacognitivos, que são denominados "funções frias"), e cita o Iowa Gambling Task (IGT) como uma das poucas situações de laboratório, até aquele momento, que consideram o aspecto da validade ecológica da tarefa.

No IGT, as escolhas, decisões e discriminação de estímulos são acompanhadas de perdas e ganhos, ou seja, o participante deve escolher entre blocos de cartas que lhe fornecerão ou retirarão valores ou pontos. $\mathrm{O}$ bloco com menor valor de reforço oferece menor valor de perdas e, ao final, o jogador obtém maior recompensa. O outro bloco oferece prêmios com maiores valores, mas o jogador que escolhe esse bloco tende a perder mais vezes e em maiores quantidades. Estudos com pacientes com lesões frontais e pré-frontais, demonstram que eles apresentam persistência na escolha do bloco de cartas desvantajoso, indicando o envolvimento dessas regiões cerebrais em comportamentos que envolvem controle inibitório. Em geral, lesões nas regiões frontais afetam de alguma maneira o desempenho dos sujeitos nos testes que tradicionalmente avaliam a capacidade de inibir respostas (ou o comportamento), auxiliando a corroborar essa relação (ALVAREZ, \& EMORY, 2006; ARDILA, 2008; GILBERT, \& BURGESS, 2008).

Nesse sentido, António Damásio em sua obra O Erro de Descartes, publicada originalmente em 1994, já havia considerado que os fatores cognitivos e emocionais são indissociáveis. De acordo com esse autor, as emoções estão ligadas aos pensamentos e ações das pessoas e os marcadores somáticos atuam como sinalizadores que modulam e antecipam nossas ações. Assim, a avaliação emocional auxilia na antecipação das consequências negativas na tomada de decisão (DAMÁSIO, 1994). 
Nos seus estudos, Damásio verificou que as respostas fisiológicas emocionais estão ausentes em pacientes com lesões orbitofrontais, em comparação com pacientes com lesões não-frontais, diante de cenas críticas com conteúdo emocional. Ele realizou um experimento utilizando sensores capazes de mensurar a resposta galvânica da pele (RGP) (um indicador fisiológico que aumenta em reação a estímulos emocionalmente eliciadores) e verificou que a RGP dos pacientes com lesão frontal não se alterou durante o processo de tomada de decisão para classificar cenas críticas, quando comparada com cenas emocionalmente neutras Tal observação subsidiou o desenvolvimento da Teoria do Marcador Somático, que propõe que as emoções são mediadas por respostas fisiológica processadas de maneira inconsciente pelo organismo, mediante a apresentação do estímulo eliciador (DAMÁSIO, 1994).

Outro aspecto importante no estudo do lobo frontal e FEs envolve a avaliação dessas habilidades ao longo da vida. Em sua revisão meta-analítica sobre FEs em adultos, Alvarez \& Emory (2006) apontam que a idade é um fator significativo no desempenho dessas funções. Assim, a sessão a seguir apresenta um panorama geral do desenvolvimento dos lobos frontais e sua relação com o FE.

\section{DESENVOLVIMENTO DO LOBO FRONTAL E SUA INFLUÊNCIA NAS FES}

O cérebro humano vai se especializando ao longo da vida. Partindo da observação de infantes em diversas faixas etárias, percebe-se que, quanto mais novo for o bebê, maior sua impulsividade, ou seja, menor a capacidade dele agir com planejamento direcionado a um objetivo e de retardar o reforço imediato para alcançar um reforço tardio (BARKLEY, 2001). Isso se deve ao fato de o lobo frontal ser a última região do cérebro a se desenvolver e, por isso, é comum se observar uma maior dificuldade em controle inibitório em crianças e adolescentes, quando comparados com em adultos.

Ao longo da primeira infância, essas habilidades mostram-se mais desenvolvidas que nos primeiros anos de vida, embora ainda pareçam limitadas. O crescente aumento na habilidade de representações abstratas da criança e de reflexões sobre suas próprias ações, acompanha o aumento do controle consciente dos pensamentos, da habilidade de regular seu próprio comportamento, de seguir regras e, consequentemente, de planejar e resolver problemas (BLAKEMORE, \& CHOUDHURY, 2006; ZELAZO, \& CUNNINGHAM, 2010).

Tanto para o desenvolvimento cognitivo quanto para a aquisição de funções cognitivas superiores, mudanças estruturais do cérebro são necessárias (STUSS, \& LEVINE, 2002; BROWN ET al., 2004), e elas estão diretamente relacionadas aos processos de sinaptogênese (formação e proliferação de novas sinapses), fortalecimento e apoptose (poda neuronal). As podas, por sua vez, 
promovem ativação mais focalizadas de áreas cerebrais, necessárias para o processamento de determinados estímulos e para a manifestação de comportamentos específicos em determinadas fases da vida (GROSSMANN, \& JOHNSON, 2007; JOHNSON ET al., 2005).

$\mathrm{O}$ amadurecimento cerebral obedece uma cronologia filogeneticamente determinada, em que as regiões ventrais do cérebro, respectivas às funções mais básicas e primitivas do comportamento humano, desenvolvem-se primeiro - se as compararmos com o processo maturacional das áreas corticais. Diversos estudos confirmam a influência das experiências e dos estímulos recebidos do ambiente nesse processo (BROWN ET AL., 2004; GIEDD ET AL., 1999; SISK, \& FOSTER, 2004; STEINBERG, 2005; TOGA, THOMPSON, \& SOWELL, 2006).

Espinet, Anderson, \& Zelazo (2012) demonstram que é possível alcançar melhora nas FEs de crianças que perseveraram nos erros durante a tarefa Dimensional Change Card Sort (DCCS) através do treino de reflexão sobre as regras da tarefa e suas causalidades, e essa melhora é acompanhada de alterações no padrão de ativação cortical. A literatura destaca, também, a capacidade vitalícia do cérebro de aprender e se adaptar ao ambiente, indicando que o processo de desenvolvimento cerebral é dinâmico e pode ocorrer em diferentes etapas da vida (BLAKEMORE, \& FRITH, 2005).

O nascimento e a puberdade são dois momentos importantes no processo da sinaptogênese, sendo tanto considerados como um período crítico para a formação cerebral e desenvolvimento cognitivo, quanto uma janela de oportunidades. Na infância, o processo inclui o cérebro como um todo; já na puberdade, a sinaptogênese afeta apenas a camada subgranular do córtex pré-frontal, indicando que o desenvolvimento desta área ainda encontra-se em curso (BLAKEMORE, \& CHOUDHURY, 2006).

A sinaptogênese é seguida de poda neuronal e reorganização cortical, possibilitando a especialização cerebral. Esse processo vai se aprimorando após a puberdade, fase marcada por mudanças hormonais, emocionais e cognitivas (BLAKEMORE, \& CHOUDHURY, 2006). Assim, nesta fase, há um aumento de conexões sinápticas nos lobos frontais que envolvem uma circuitaria complexa e que otimiza processos cognitivos como abstração, elaboração de hipóteses, resolução de problemas mais complexos, flexibilização cognitiva e inibição de respostas automáticas e preponderantes (KOLB, \& WHISHAW, 2002).

Através de estudos longitudinais com imagem por ressonância magnética observa-se que o crescimento da substância cinzenta (conjunto de corpos neuronais) durante a adolescência acontece de maneira não linear, regional e temporal, ao passo que regiões com funções sensoriais amadurecem antes que regiões relacionadas a aspectos cognitivos e emocionais (GIEDD ET AL., 1999; GOGTAY ET AL., 2004). Desse modo, entende-se que o processo maturacional do cérebro está vinculado à maturação emocional e cognitiva do adolescente (BLAKEMORE, \& CHOUDHURY, 2006). 


\section{FES E SUAS CONEXÕES ANATÔMICAS E FUNCIONAIS}

Os estudos de imageamento cerebral e de ativação cortical demonstram que a relação entre disfunções executivas e o lobo frontal não é linear, pois outras regiões corticais, subcorticais e posteriores também estão envolvidas na rede que sustenta essas funções (ARDILA, 2008).

O córtex pré-frontal estabelece fortes conexões com o córtex sensorial e com o córtex motor, e com estruturas subcorticais, como os gânglios da base (GILBERT, \& BURGESS, 2008). Mapeando essas interconexões e esses circuitos funcionais, verifica-se que, didaticamente, a região frontal pode ser dividida em três sub-regiões quanto ao papel das FEs. O córtex frontal dorsolateral (CFDL) (ALVAREZ, \& EMORY, 2006) e córtex pré-frontal dorsolateral (CPFDL) (ARDILA, 2008; GILBERT, \& BURGESS, 2008) apresentam correlações com FEs metacognitivas ou frias, como as de planejamento, memória operacional, pensamento abstrato e resolução de problemas (ALVAREZ, \& EMORY, 2006; ARDILA, 2008; GILBERT, \& BURGESS, 2008).

Já o córtex pré-frontal ventromedial (CPFVM), relacionado às FEs quentes, estabelece fortes conexões com o sistema límbico, e seu papel está ligado ao controle inibitório de impulsos e à inibição de comportamentos instintivos em função do contexto em que se inserem (ARDILA, 2008). Lesões nessa região provocam apatia, diminuição de interação social e retardo psicomotor (ALVAREZ, \& EMORY, 2006).

A terceira região, o córtex orbitofrontal (COF) também está diretamente relacionado às funções de controle inibitório e aos comportamentos sociais. Essa região está implicada na inibição de entradas sensoriais internas e externas que podem interferir na manifestação de algum comportamento que já esteja sendo executado (ALVAREZ, \& EMORY, 2006; ARDILA, 2008). Lesões nessa região estão ligadas à impulsividade e comportamentos antissociais.

Em algumas espécies animais, cujo sistema nervoso também aprende por contingências de reforçamento, o comportamento impulsivo pode não se manifestar como um problema, na medida que ele é modelado por consequências imediatas. Na verdade, pode representar um mecanismo de defesa, vinculado ao sistema autônomo de luta ou fuga. No entanto, no ser humano, o controle do impulso apresenta-se como um mecanismo adaptativo e um pré-requisito para o desenvolvimento de funções superiores que exigem autocontrole ou ainda considerar consequências não-imediatas como reforçadores (BARKLEY, 2001).

Verifica-se, então, que o córtex pré-frontal (CPF) está fortemente relacionado com os papéis de controle e monitoramento de estímulos, coordenando a relação entre cognição e emoção. Considera-se que a principal função dessa região é controlar os impulsos do sistema límbico, tornando o comportamento social e moralmente aceitável (ARDILA, 2008). 
Ardila (2008), fundamentado por evidências anatômicas e clínicas do lobo pré-frontal, propõe que o principal papel das FEs metacognitivas é a representação mental das ações, ideia esta que vem ao encontro da teoria de Vygotsky acerca do papel da linguagem no desenvolvimento das funções mentais superiores (VYGOTSKY, 1989). Vygotsky propõe que o pensamento está associado ao processo de internalização da fala, ou seja, um comportamento motor encoberto. Ardila (2008) corrobora Vygotsky (1989), acrescentando que o córtex pré-frontal, evolutivamente, derivou-se do córtex frontal, onde se localizam a área motora e também a área motora da fala. Além disso, lesões pré-frontais que atingem o desempenho metacognitivo também desencadeiam distúrbios motores.

No processo de evolução motora dos hominídeos, é possível perceber que o substrato neural responsável pelo controle motor - a saber, os gânglios da base, o cerebelo e o córtex frontal desenvolveu-se de modo a oferecer suporte para o aparato cognitivo e linguístico atual. Além dessas evidências, a descoberta dos neurônios-espelho (aqueles que se ativam quando uma pessoa vê outra desempenhando uma ação motora) por Giacomo Rizolatti e sua equipe em 1996 reforçam ainda mais a ideia da influência da internalização do comportamento motor no desenvolvimento da linguagem e, consequentemente, das funções cognitivas superiores (KILNER, \& BLAKEMORE, 2007). Essa estrutura permite o desenvolvimento de habilidades sociais como o reconhecimento, a compreensão e a previsão de consequências de ações do próximo.

Pode-se supor que na pré-história as habilidades metacognitivas não se mostravam tão necessárias à sobrevivência da espécie como ocorre hoje. Porém, o uso de estratégias sociais e a convivência em grupo são fatores que facilitaram a sobrevivência da espécie humana, estimulando o uso e ativação das regiões cerebrais envolvidas nesse processo - os lobos frontais - emergindo os processos atualmente relacionados ao funcionamento executivo emocional (ARDILA, 2008). Sistemas adaptativos emergem para resolver problemas adaptativos, e a evolução é a explicação com maior credibilidade, até o momento, para subsidiar a compreensão desses processos (Barkley, 2001).

\section{CONSIDERAÇÕES FINAIS}

Há algumas décadas verificava-se forte ênfase nas FEs como funções apenas metacognitivas, sendo que instrumentos de pesquisas foram desenvolvidos para mensurar-se esse aspecto isolando-se o sujeito do contexto social, e as emoções de outros aspectos de sua cognição. No entanto, evidências neurocientíficas acerca da relação entre estruturas corticais e subcorticais indicam que o funcionamento cognitivo humano é fortemente ligado ao funcionamento emocional.

As funções cognitivas superiores, as FEs, a linguagem verbal e os mecanismos de controle cognitivos ganham destaque como comportamentos tipicamente humanos. Esse conjunto de 
habilidades oferece um aparato propício para a convivência social, para o planejamento de ações e para atividades que envolvam mentalização. Nesse sentido, a compreensão do funcionamento cerebral mostra-se fundamental a todos aqueles que desejam entender o homem como um ser evolutivo. Tanto os estudos filogenéticos como aqueles que enfocam o desenvolvimento do homem a cada geração têm mostrado a necessidade de integração dos elementos emocionais, cognitivos e biológicos para a compreensão do funcionamento cerebral, possibilitando aplicações para pessoas com ou sem lesões, com desenvolvimento normal ou com déficits executivos e em áreas afins, como educação e saúde.

\section{REFERÊNCIAS}

ALVAREZ, Julie A.; EMORY, Eugene. Executive function and the frontal lobes: a meta-analytic review. Neuropsychology review, v. 16, n. 1, p. 17-42, 2006.

ARDILA, Alfredo. On the evolutionary origins of executive functions. Brain and cognition, v. 68, n. 1, p. 92-99, 2008.

BARKLEY, Russell A. The executive functions and self-regulation: An evolutionary neuropsychological perspective. Neuropsychology review, v. 11, n. 1, p. 1-29, 2001.

BLAKEMORE, Sarah-Jayne; CHOUDHURY, Suparna. Development of the adolescent brain: implications for executive function and social cognition. Journal of child psychology and psychiatry, v. 47, n. 3-4, p. 296-312, 2006.

BLAKEMORE, Sarah-Jayne; FRITH, Uta. The learning brain: lessons for education: a précis. Developmental science, v. 8, n. 6, p. 459-465, 2005.

BROWN, Timothy T. et al. Developmental changes in human cerebral functional organization for word generation. Cerebral Cortex, v. 15, n. 3, p. 275-290, 2005.

CAPOVILLA, Alessandra Gotuzo Seabra; ASSEF, Ellen Carolina dos Santos; COZZA, Heitor Francisco Pinto. Avaliação neuropsicológica das funções executivas e relação com desatenção e hiperatividade. Avaliação psicológica, v. 6, n. 1, p. 51-60, 2007.

DAMÁSIO, ANTÓNIO. Descartes' error: Emotion, reason and the human brain, Putnan Pub Group, 1994.

ESPINET, Stacey D.; ANDERSON, Jacob E.; ZELAZO, Philip David. Reflection training improves executive function in preschool-age children: Behavioral and neural effects. Developmental cognitive neuroscience, v. 4, p. 3-15, 2013. 
FOURNIER-VICENTE, Siobhan; LARIGAUDERIE, Pascale; GAONAC'H, Daniel. More dissociations and interactions within central executive functioning: A comprehensive latent-variable analysis. Acta psychologica, v. 129, n. 1, p. 32-48, 2008. Artigo disponível em: $<$ http://www.sciencedirect.com/science/article/pii/S0001691808000565>. Acesso em: 23 jun. 12.

GILBERT, Sam J.; BURGESS, Paul W. Executive function. Current Biology, v. 18, n. 3, p. R110R114, 2008.

GIEDD, Jay N. et al. Brain development during childhood and adolescence: a longitudinal MRI study. Nature neuroscience, 1999. v. 2, n. 10, p. 861-863.

GOGRAY, N. et al. Dynamic mapping of human cortical development during childhood through early adulthood. Proceedings of the National Academy of Science of the United States of America, 2004. 101(21), pp. 8174-8179.

GOLDSTEIN, Sam et al. Introduction: a history of executive functioning as a theoretical and clinical construct. In: Handbook of executive functioning. Springer, New York, NY, 2014. p. 3-12.

GROSSMANN, Tobias; JOHNSON, Mark H. The development of the social brain in human infancy. European Journal of Neuroscience, v. 25, n. 4, p. 909-919, 2007.

JOHNSON, Mark H. et al. The emergence of the social brain network: Evidence from typical and atypical development. Development and psychopathology, v. 17, n. 03, p. 599-619, 2005. Disponível em: <http://www.blackwell-synergy.com/doi/abs/10.1111/j.1469-7610.2004.00320.x>. 15 dez. 2012.

KILNER, James M.; BLAKEMORE, Sarah-Jayne. How does the mirror neuron system change during development?. Developmental science, v. 10, n. 5, p. 524-526, 2007. Disponível em: $<$ http://www.ncbi.nlm.nih.gov/pubmed/17683337>. Acesso em: 15 out. 2012.

KOLB, Brian; WHISHAW, I.Q. Neurociência do Comportamento. Barueri: Manole, Cap: 11, 398443, 2002.

LEZAK, Muriel Deutsch. Neuropsychological assessment. Oxford university press, 2004. Fourth Edition. Oxford University Press.

LEZAK, Muriel D.; O'BRIEN, Kevin P. Longitudinal study of emotional, social, and physical changes after traumatic brain injury. Journal of Learning Disabilities, v. 21, n. 8, p. 456-462, 1988. 
MIGUEL, Fabiano Koich. Teste Wisconsin de Classificação de Cartas. 2005.

MIYAKE, Akira et al. The unity and diversity of executive functions and their contributions to complex "frontal lobe" tasks: A latent variable analysis. Cognitive psychology, v. 41, n. 1, p. 49$100,2000$.

MIYAKE, Akira; FRIEDMAN, Naomi P. The nature and organization of individual differences in executive functions four general conclusions. Current directions in psychological science, v. 21, n. 1, p. 8-14, 2012.

PRIGATANO, George P. Personality disturbances associated with traumatic brain injury. Journal of Consulting and Clinical Psychology, v. 60, n. 3, p. 360, 1992.

SISK, Cheryl L.; FOSTER, Douglas L. The neural basis of puberty and adolescence. Nature neuroscience, v. 7, n. 10, p. 1040-1047, 2004.

STEINBERG, Laurence. Cognitive and affective development in adolescence.Trends in cognitive sciences, v. 9, n. 2, p. 69-74, 2005.

STUSS, Donald T.; LEVINE, Brian. Adult clinical neuropsychology: lessons from studies of the frontal lobes. Annual review of psychology, v. 53, n. 1, p. 401-433, 2002.

TOGA, Arthur W.; THOMPSON, Paul M.; SOWELL, Elizabeth R. Mapping brain maturation. Focus, 2006.

VYGOTSY, Lev S. et al. Pensamento e linguagem. Edição eletrônica: Ed Ridendo Castigat Mores. Disponível em, 1989.

KERR, Aurora; ZELAZO, Philip David. Development of "hot" executive function: The children's gambling task. Brain and cognition, v. 55, n. 1, p. 148-157, 2004.

ZELAZO, Philip David; CUNNINGHAM, William A. Executive Function: Mechanisms Underlying Emotion Regulation. 2007. Disponível em: $<$ http://faculty.psy.ohiostate.edu/cunningham/documents/Zelazo_Cunningham_2007.pdf $>$. Acesso em: 15 out. 2012.

$\begin{array}{ll}\text { Histórico } & \text { Recebido em: 3-12-2015 } \\ & \text { Revisado em: 4-8-2018 } \\ & \text { Aceito em: 4-9-2018 }\end{array}$

